\title{
Millimeter-Wave Offset Fresnel Zone Plate Lenses Characterization
}

\author{
Germán León ${ }^{1,}{ }^{*}$, Luis F. Herrán ${ }^{1}$, Max Munoz $^{2}$, Fernando Las-Heras ${ }^{1}$, and Yang Hao ${ }^{2}$
}

\begin{abstract}
Fresnel Zone Plate Lenses (FZPLs) are transparent-opaque lenses that filter the desirable phase. The centred Fresnel lenses have a strong back radiation towards the feed. In order to solve this drawback, offset feeding or offset pointing lenses are used. In this work, both offset FZPLs are studied using an optical physics method and experimentally characterized in the millimeter band. Two prototypes have been manufactured and measured, presenting a narrow beamwidth. The characteristics of pointing of this beam are studied depending on the feed gain. This work shows the pointing characteristics of the lenses, simply moving the lens in a plane.
\end{abstract}

\section{INTRODUCTION}

Fresnel Zone Plate Lenses (FZPLs) have been used in a wide range of the electromagnetic spectrum, from microwave [1,2] to X-rays [3]. They are transparent-opaque lenses that filter the desire phase of the impinging wave, reflecting the undesirable phase and transforming a spherical front phase in a plane one. These lenses keep on being of interest in antenna applications, both in Far Field (FF) [4-7] and in Near Field (NF) [8], due to their simplicity to manufacture and lower weight and profile when compared to classical dielectric lenses.

The FZPLs present a narrowband [9], strong back-radiation and therefore poor efficiency, which is usually below 20\%. Phase-correcting FZPLs [10] improves the efficiency, replacing the transparentopaque zones by different permittivity dielectrics zones [11] or different grooved zones [12]. An alternative to FZPLs, based on a planar periodic screen at optical frequencies, has been presented [13], although, following the authors, improvement on the efficiency has still to be done.

All these works focus on centred FZPL and very few analyses can be found on offset FZPL $[14,15]$. The offset FZPLs have two advantages. First, the back-radiation is not directed to the source and mismatching is reduced. Second, the feed residual radiation is not focused to the same direction of the main beam. In the NF, this characteristics is exploited to produce a plane wave with the required amplitude and phase variation. These lenses has been used in Compact Antenna Test Ranges (CATR) $[16,17]$ at millimeter and sub-millimeter frequencies because of their simplicity to manufacture and relatively good tolerances at these frequencies. These lenses have been optimized and used in CATR measurement at sub-millimeter frequencies [18-20].

This work presents a study of the beam-scanning capability of these lenses and their potential application to imaging systems.

\section{OFFSET FZPL DESCRIPTION}

The goal of the FZPL sketched in Figure 1(a) is to convert a spherical wave into a plane wave front whose propagation vector forms an angle of $\theta_{0}$ degrees with the $Z$-axis. This scheme also shows that

\footnotetext{
Received 19 September 2014, Accepted 17 October 2014, Scheduled 3 November 2014

* Corresponding author: Germán León (gleon@uniovi.es).

1 Signal and Communications Group, Electrical Engineering Department, Universidad de Oviedo, Spain. ${ }^{2}$ School of Electronic Engineering and Computer Science, Queen Mary University of London, England.
} 
the residual radiation of the system has the same direction of the radiation of the feed and that the back radiation forms an angle of $\theta_{0}$ with the $Z$-axis.

The Fresnel zones in a general case are formed by ellipses $[1,14]$. The $n$ th-ellipse limits the phase of the spherical wave, between $(n-1) \cdot \pi$ and $n \cdot \pi$. These ellipses are described by the equations:

$$
\begin{aligned}
& \left(\frac{x-C_{n}}{A_{n}}\right)^{2}+\left(\frac{y}{B_{n}}\right)^{2}=1 \\
& A_{n}=\frac{\sqrt{n \lambda\left(F \cos ^{2} \theta_{0}+\frac{n \lambda}{4}\right)}}{\cos ^{2} \theta_{0}} \\
& B_{n}=\left|\cos \theta_{0}\right| A_{n} \\
& C_{n}=\frac{n \lambda \sin \theta_{0}}{2 \cos ^{2} \theta_{0}}
\end{aligned}
$$

where $\lambda$ is the wavelength; $F$ is the focal distance; $A_{n}$ and $B_{n}$ are the semi-axis of the ellipses in the $X$ and $Y$ axes; $C_{n}$ is the position along the $X$-axis of the centre of the $n$ th-ellipse.

Transparent-opaque rings are interspersed in order to the desirable phase filter. Although it is indifferent the type of the initial ring because they are complementary surfaces, in the case of the lenses, the first ellipse is usually chosen as transparent.

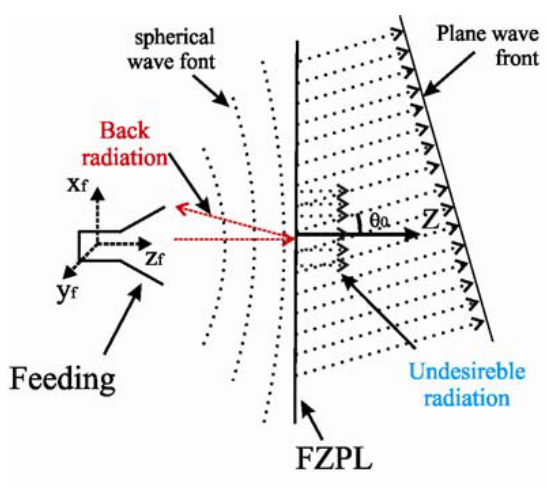

(a)

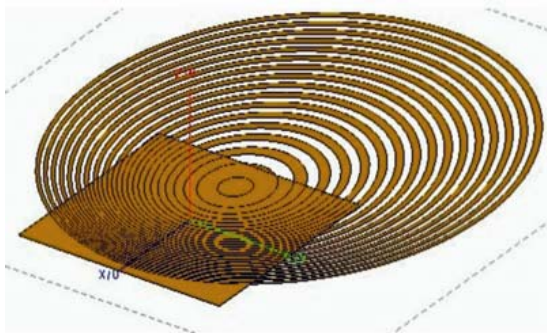

(b)

Figure 1. (a) Scheme behaviour of an offset FZPL pointing towards $\theta_{0}$ with respect to $Z$-axis. (b) Layout of a FZPL, designed to point towards $20^{\circ}$.

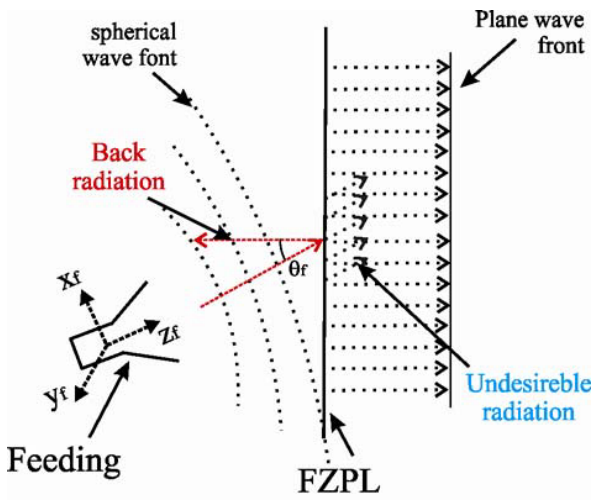

(a)

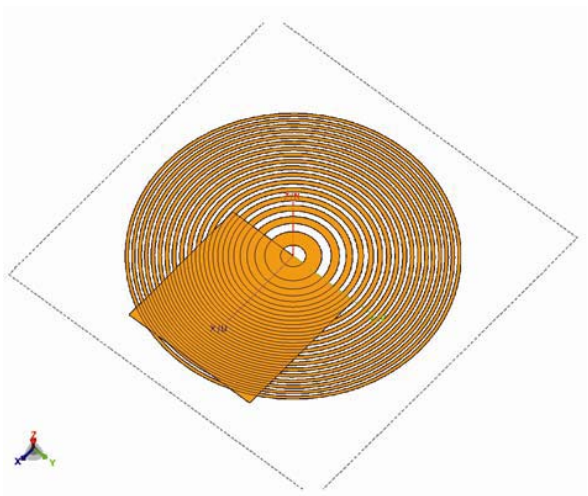

(b)

Figure 2. (a) Scheme behaviour of an offset feed FZPL pointing at $Z$-axis. (b) Layout of an offset FZPL, with $\theta_{f}=38^{\circ}$. 
Figure 1(b) shows the ellipses in the specific lens when $\theta_{0}=20^{\circ}, F=80 \mathrm{~mm}$ and a frequency of $95 \mathrm{GHz}$. A centred square indicates the piece of the lens to be simulated.

It is clear that if $\theta_{0}=0$, the ellipses turn into circles (Figure 2(a)), and the FZPL is centred one. In this case, the offset lens is easy to obtain, choosing a non-centred surface to manufacture (Figure 2(b)) and pointing the feed to the centre of the lens. In this case, the feed forms an angle of $\theta_{f}=38^{\circ}$ with the $Z$-axis, the same as the residual radiation, and the back radiation is directed to $-180^{\circ}$, maintaining the benefits of an offset FZPL.

The radiation patterns of these two lenses, fed by a point source, have been simulated by means of Physical Optic (PO) techniques using a commercial simulation software tool [21]. In this way, a centred FZPL, with the same $F$, size and feed has been simulated, to compare (Figure 3) advantages of the offset ones. The radiation patterns of the three sets present two narrow main beams, one in the forward direction and the other to the back direction. The back-radiation of the centred lens points to $180^{\circ}$, which is where the source is placed. This backradiation can produce mismatch, when a real feed is used. The back-radiation of the offset feed FZPL points also towards $180^{\circ}$, but this does not correspond to the feed direction. The back-radiation of the $20^{\circ}$ pointing FZPL is directed to $-160^{\circ}$ and will not affect the behaviour of a real feed.

These three radiation patterns also exhibit undesirable radiation in the same direction the feed points to. In the offset case, this radiation does not affect the main beam in the forward direction. However, in the centred lens, the undesirable radiation and the main beam have the same direction, and a distortion of the main beam can be produced.

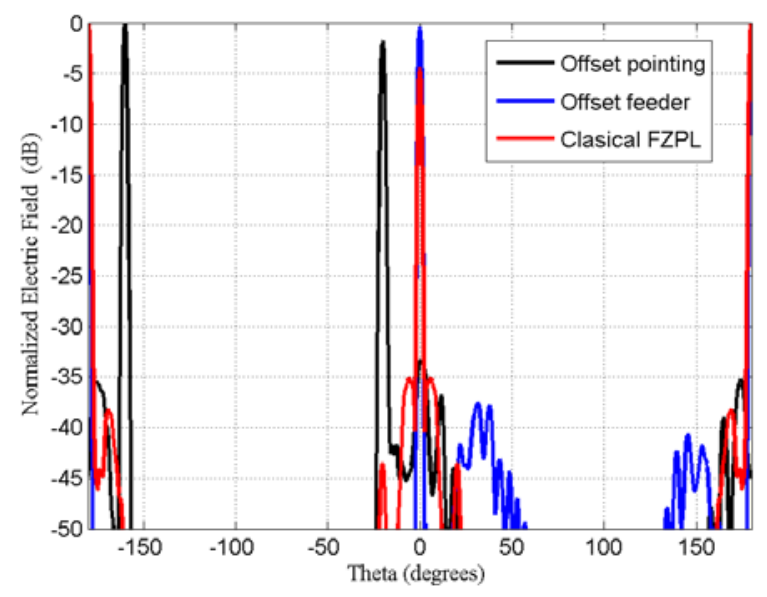

Figure 3. Radiation pattern of three FZPLs: a $20^{\circ}$ pointing, an offset feeding and a centred lens.

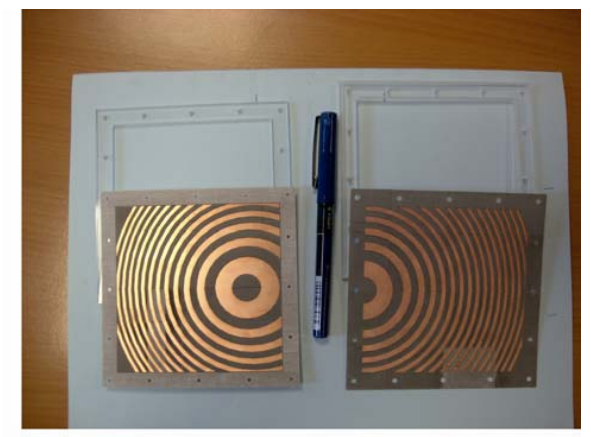

(a)

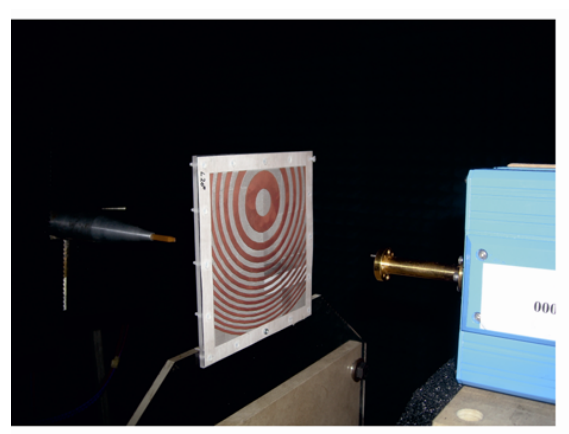

(b)

Figure 4. (a) Manufactured FZPLs. (b) Picture of the lenses measurement set-up. 


\section{SIMULATED AND MEASURED RESULTS}

In this work, the aforementioned lenses have been manufactured on an 8 mils RO4003 substrate (Figure 4(a)). Both are square lenses with a side of $100 \mathrm{~mm}$ and a centred focal distance $F=80 \mathrm{~mm}$. The analysis of these lenses has been done using PO approximation. In the simulations, the metal strips are supposed to be suspended on air, neglecting the thickness of the substrate. This approximation has demonstrated to be accurate enough in the NF [8].

The lenses, which rest on a methacrylate frame, have been measured in a mm-wave planar scanner in the facilities of the Queen Mary University of London (Figure 4(b)).

An open waveguide (OWG) and a $20 \mathrm{~dB}$ gain horn antenna have been considered as feeds (Figure 5). The more directive feed produces a strong undesirable radiation in the feed pointing direction, so the OWG has been chosen due to its less directive radiation pattern, although spillover radiation increases. The edge illumination with the OWG around $-12 \mathrm{~dB}$.

\subsection{Offset Pointing Lens}

Figure 6 represents the simulated and measured results for a $20^{\circ}$ pointing FZPL. The system, made up by the feed and the lens, exhibits a $-3-\mathrm{dB}$ beamwidth lower than three degrees. This picture presents the ability of beam scanning of the lens. The simple movement of the feed in the $Y$-axis $\left(x_{f}=0\right.$, $\left.y_{f}=15 \mathrm{~mm}\right)$ produces a change in the pointing direction of more than $\pm 10^{\circ}$. The scanning capability could be increased, but the secondary lobes level would increase. In these three cases of study, simulated and measured results agree very well in the main beam, validating the approximations and simulation method for the main propagation contribution. The measurements present a higher radiation level out of the main beam due to the diffraction generated by the lens frame, diffraction which is not taken into account in the model.

These results have also been represented in Figure 7, where the beam scanning ability of this lens is studied. Simulated and measured $U-V(U=\sin \theta \cos \phi, V=\sin \theta \cos \phi)$ patterns are plotted in this picture, using continuous line for the $-3 \mathrm{~dB}$ beam contour and dashed lines for the $-10 \mathrm{~dB}$ beam contour. In all cases, simulated and measured results matched very well. For $y_{f}=0$, the parameter $x_{f}$ has been modified and an increasing of the $-3 \mathrm{~dB}$ beamwidth can be found. In this case, up to $\pm 15^{\circ}$ of scanning capability can be found.

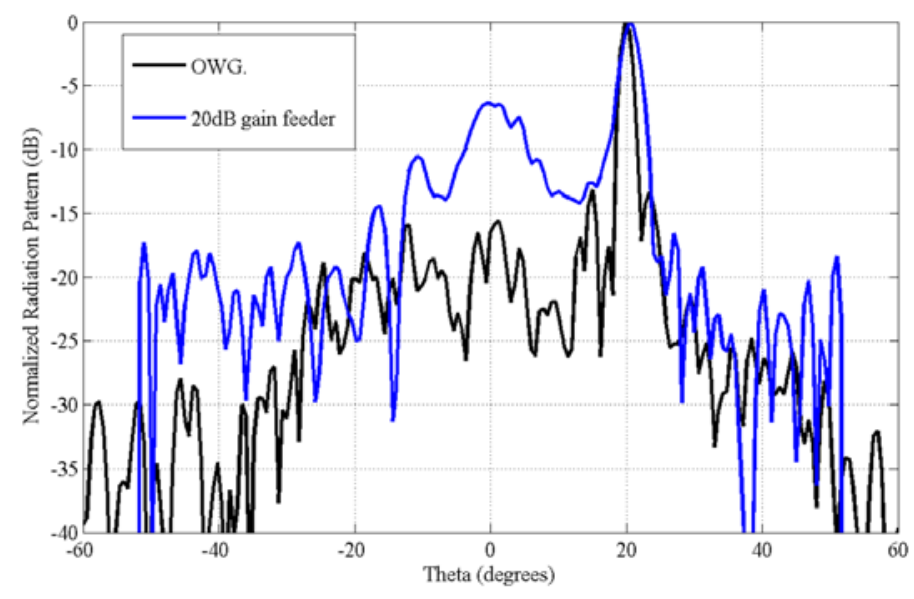

Figure 5. Radiation pattern produces by the configuration set by the FZPL and an OWG (black) or a $20 \mathrm{~dB}$ gain horn antenna (blue).

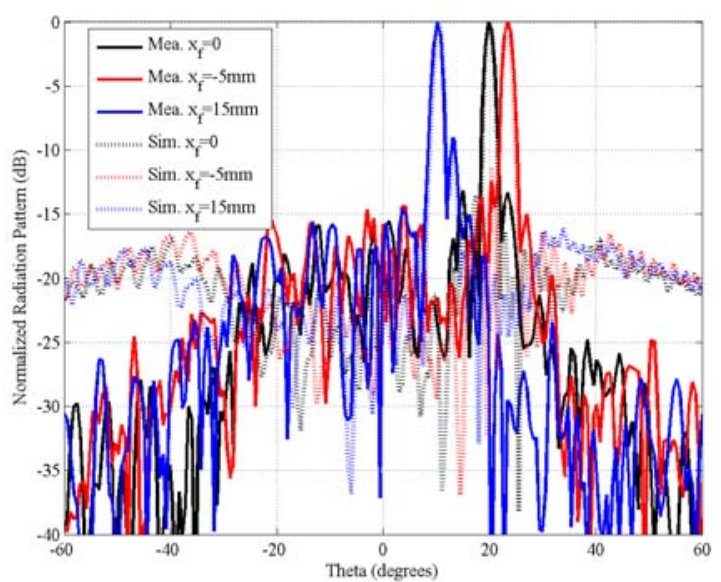

Figure 6. Radiation pattern of the $20^{\circ}$ pointing FZPL for different feed location. 


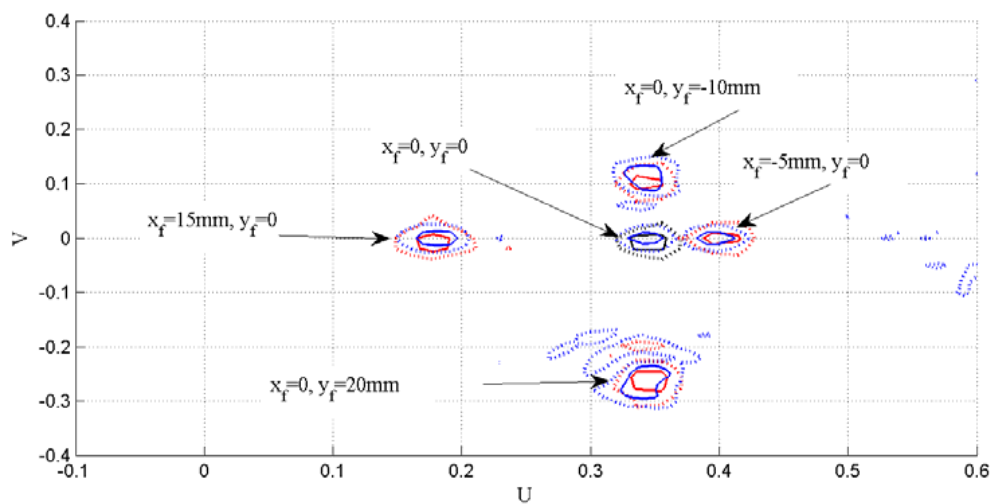

Figure 7. Contour $U-V$ plot of the scanning ability of the $20^{\circ}$ pointing FZPL. Simulated results are in blue colour and measured results are in black and red, continuous lines for $-3 \mathrm{~dB}$ contours and dashed lines for $-10 \mathrm{~dB}$ contours.

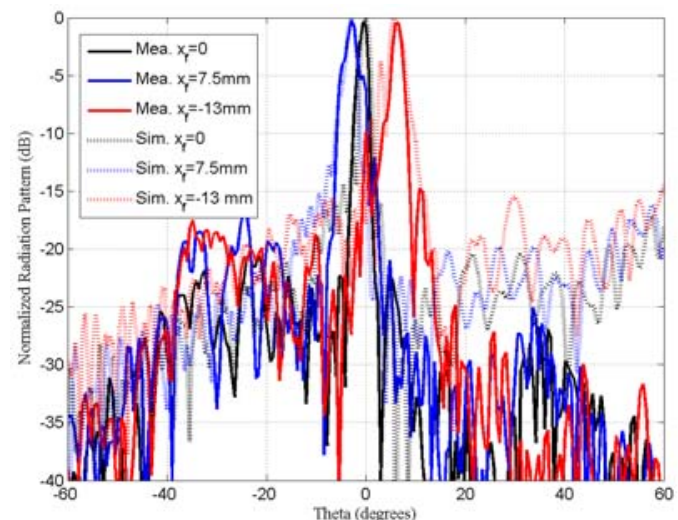

Figure 8. Radiation pattern of the offset feed FZPL for different feed location.

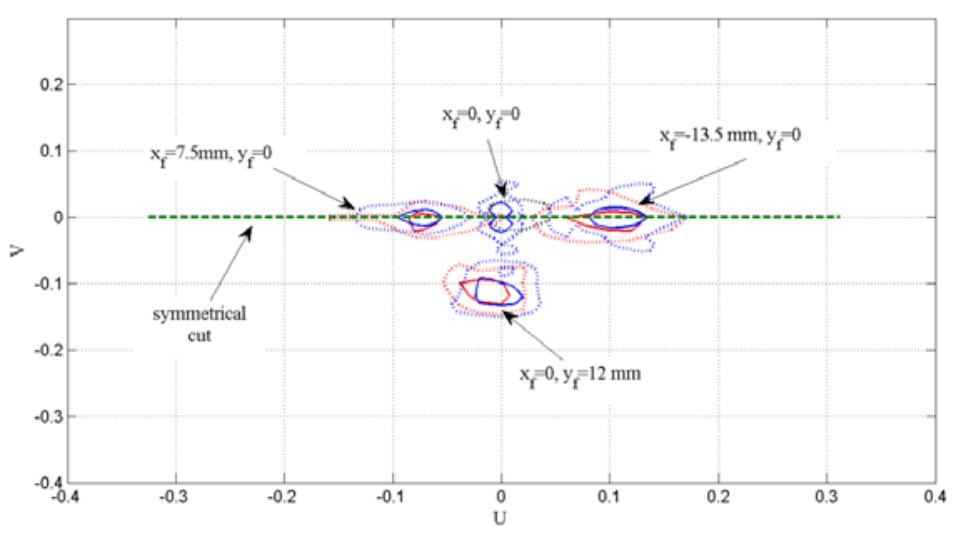

Figure 9. Contour U-V plot of the scanning ability of the offset feed FZPL. Simulated results are in blue colour and measured results are in black and red, continuous lines for $-3 \mathrm{~dB}$ contours and dashed lines for $-10 \mathrm{~dB}$ contours.

\subsection{Offset Feed Set}

An offset feed FZPL has also been studied (Figure 8). This lens points to $0^{\circ}$ with a $-3 \mathrm{~dB}$ beam width of about $3^{\circ}$. The configuration presents a lower sensitivity than the last lens. Figures 8 and 9 show that a feed displacement along the $Y$-axis produces a decrease of beam scan capability and an increase of the beamwidth, when compared to the case of $20^{\circ}$ pointing lens.

\section{CONCLUSIONS}

This work focuses on an experimental study of two offset FZPLs that try to overcome some drawbacks of the centred FZPLs. One lens points to a $20^{\circ}$ direction and the other is an offset feed lens. Both lenses avoid mismatch of the feed because backradiation is not directed to the source localization. Good agreement has been found between simulations via PO method and experimental results. This work studies the behaviour of the radiation pattern of the configuration set by the feed and the lens when the feed shifts. The $20^{\circ}$ direction lens presents a good scanning properties, with more than $10^{\circ}$ in the principal directions. However, the offset feed lens presents lower scanning ability. 


\section{ACKNOWLEDGMENT}

Germán León would like to thank to Mrs Isabel Martín for encouraging him to work in this topic. Also, this author would like to express his gratitude to Mrs. Laura García-Perales, Dr. Oscar Quevedo-Teruel and Dr. Rhiannon Mitchell-Thomas for their generous help during the measurement campaign.

This work was supported by the Spanish Ministry of Economy and Competitiveness under the project TEC2011-24492 "Inverse scattering techniques for imaging: new approaches and measurements techniques-iScat", CSD2008-00068 "Teraherrtz technology for electromagnetic sensing applications: teratense" and by an excellent mobility grant financed by the University of Oviedo and the city council of Gijón.

\section{REFERENCES}

1. Hristov, H. D., Fresnel Zones in Wireless Links, Zone Plate Lenses, and Antennas, Artech House, Boston, MA, 2000.

2. Minin, O. V. and I. V. Minin, Diffractional Optics of Millimeter Waves, Institute of Physics, Publishing, Bristol, UK, 2004.

3. Yun, W. B., "Mossbauer-Fresnel zone plate," Journal of Applied Physics, Vol. 71, No. 11, 57095711, 1992.

4. Stouy-Grandy, S. M., A. Petosa, I. V. Minin, O. V. Minin, and J. Wight, "A systematic study of varying reference phase in the design of circular Fresnel zone plate antenna," IEEE Trans. Antennas Propag., Vol. 54, No. 12, 3629-3637, Dec. 2006.

5. Reid, D. R. and G. S. Smith, "A full electromagnetic analysis for the Soret and folded zone plate antennas," IEEE Trans. Antennas Propag., Vol. 54, No. 12, 3638-3646, Dec. 2006.

6. Webb, G. W., I. V. Minin, and O. V. Minin, "Variable reference phase in diffractive antennas: Review, applications, new results," IEEE Antennas Propag. Mag., Vol. 53, No. 2, 77-94, Mar. 2011.

7. Gallacher, T. F., D. A. Robertson, and G. M. Smith, "The photo-injected Fresnel zone plate antenna: Optoelectronic beam steering at mm-wave frequencies," IEEE Trans. Antennas Propag., Vol. 61, No. 4, 1688-1696, Apr. 2013.

8. Karimkachi, S. and A. A. Kishk, "Focusing properties of Fresnel zone plate lens antennas in the near-field region," IEEE Trans. Antennas Propag., Vol. 59, No. 5, 1481-1487, May 2011.

9. Chen, G.-S. and H.-C. Yeh, "Polarization-selective color-filter Fresnel lens in polymer-stabilized cholesteric liquicrystals," Journal of Applied Physics, Vol. 112, 054501, 2012.

10. Black, D. and J. C. Wiltse, "Millimeter-wave characteristics of phase-correcting Fresnel zone plates," IEEE Trans. Microwave Theory Tech., Vol. 35, No. 12, 1122-1129, Dec. 1987.

11. Hristov, H. D. and J. M. Rodriguez, "Design equation for multidielectric Fresnel zone plate lens," IEEE Microwave Wire. Comp. Letters, Vol. 22, No. 11, 574-576, 2011.

12. Hristov, H. D. and M. H. A. J. Herben, "Quarter-wave Fresnel zone planar lens and antenna," IEEE Microw. Guided Wave Letters, Vol. 5, No. 8, 249-251, Aug. 1995.

13. Aieta, F., P. Genevet, M. A. Kats, N. Yu, R. Blanchrad, Z. Gaburro, and F. Capasso, "Aberrationfree ultra-thin flat lenses and axicons at telecom wavelengths based on plasmonic metasurfaces," Nano Letters, Vol. 12, No. 9, 4932-4936, 2012.

14. Guo, Y. J. and S. K. Barton, "Offset Fresnel zone plate antennas," Int. J. of Satellite Communications, Vol. 12, No. 4, 381-385, 1994.

15. Van Outen, J. M. and M. H. A. J. Herben, "The elliptical Fresnel zone plate antennas," Ninth International Conference on Antennas and Propagation, (Conf. Publ. No. 407), Vol. 1, 997-101, 1995.

16. Tuovinen, J., A. Vasara, and A. V. Raisanen, "A new type of compact antenna test range," Proceedings of the 22nd European Microwave Conference, Vol. 1, 503-508, Espoo, 1992.

17. Sehm, T., J. Ala-Laurinaho, T. Hirvonen, and A. V. Räisänen, "Antenna measurement using hologram CATR," Electronics Letters, Vol. 35, No. 10, 757-758, 1999. 
18. Hirvonen, T., J. P. S. Ala-Laurinaho, J. Tuovinen, and A. V. Räisänen, "Compact antenna test range based on a hologram," IEEE Trans. Antennas Propag., Vol. 45, No. 8, 1270-1276, Aug. 1997.

19. Häkli, J., T. Koskinen, A. Lönnqvist, J. Säily, V. Viikari, J. Mallat, J. Ala-Laurinaho, J. Tuovinen, and A. V. Räisänen, "Testing of a $1.5-\mathrm{m}$ reflector antenna at $322 \mathrm{GHz}$ in a CATR based on a hologram," IEEE Trans. Antennas Propag., Vol. 53, No. 10, 3142-315, Oct. 2005.

20. Karttunen, A., J. Ala-Laurinaho, M. Vaaja, T. Koskinen, J. Häkli, A. Lönnqvist, J. Mallat, A. Tamminen, V. Viikari, and A. V. Räisänen, "Antenna tests with a hologram-based CATR at $650 \mathrm{GHz}$," IEEE Trans. Antennas Propag., Vol. 57, No. 3, 711-720, Mar. 2009.

21. FEKO Suite 6.2, Stellenbosch, South Africa, 2013. 